\title{
High speed femtosecond pulse characterization based on spectral interferometry
}

\section{Caracterización de alta velocidad de pulsos de femtosegundos basado en interferometría espectral}

\author{
G. Castro-Olvera, J. Garduño-Mejía $\left({ }^{*}, \mathrm{~A}\right), \mathrm{M}$. Rosete-Aguilar, \\ C. J. Román-Moreno ${ }^{(A)}$, R. Ortega-Martínez ${ }^{(A)}$ \\ CCADET-UNAM, Apdo, Postal 70-186, C.P. 04510. Coyoacán, Cd. Universitaria, México D.F., México. \\ (*) Email: jesus.garduno@ccadet.unam.mx A: miembro de AMO / AMO member \\ Recibido / Received: 06/09/2012. Revisado / Revised: 16/11/2012. Aceptado / Accepted: 19/11/2012. \\ DOI: http://dx.doi.org/10.7149/OPA.46.1.1
}

\begin{abstract}
:
In this work, we present spectrum and spectral phase retrieval of femtosecond pulses at $74 \mathrm{~Hz}$ refresh rate by a spectral interferometry technique. Different spectral phase designs including high order chirp are measured and generated with a pulse shaper based on a micro-machined deformable membrane mirror (MMDM).
\end{abstract}

Key words: Ultrafast Optics, Pulse Characterization, Femtosecond Pulse Shaping.

\section{RESUMEN:}

En este trabajo presentamos la recuperación del espectro y fase espectral de pulsos de femtosegundos a una tasa de repetición de $74 \mathrm{~Hz}$ por una técnica de interferometría espectral. Diferentes diseños de fase espectral incluida automodulación de fase de orden superior son medidos y generados por un reconfigurador de pulsos basado en un espejo deformable de membrana micromaquinado (MMDM).

Palabras clave: Óptica Ultrarrápida, Caracterización de Pulsos, Reconfiguración de Pulsos de Femtosegundos.

\section{REFERENCIAS Y ENLACES / REFERENCES AND LINKS}

[1]. A. Weiner, "Programmable shaping of femtosecond optical pulses by use of 128-element liquid crystal phase modulator", IEEE J. Quantum. Elect. 28, 908-920 (1992). DOI

[2]. F. Verluise, V. Laude, Z. Cheng, Ch. Spiemann, P. Tournois, "Amplitude and phase control of ultrashort pulses by use of an acousto-optic programmable dispersive filter: pulse compression and shaping", Opt. Lett. 25, 575-577 (2000). DOI

[3]. J. Garduño-Mejía, A. H. Greenaway, D. T Reid, "Designer femtosecond pulses using adaptive optics", Opt. Express 11, 2030-2040 (2003). DOI

[4]. J. Garduño-Mejía, E. Ramsay, A. Greenaway, D. T. Reid, "Real time femtosecond optical pulse measurement using a video rate FROG system”, Rev. Sci. Instrum. 74, 3624-3627 (2003).

[5]. T. M. Shuman, M. E. Anderson, J. Bromage, C. Iaconis, L. Waxer, I. A. Walmsley, "Real-time SPIDER: ultrashort pulse characterization at $20 \mathrm{~Hz}$ ", Opt. Express 5, 134-143 (1999). DOI

[6]. C. Dorrer, F. Salin, "Characterization of spectral phase modulation by classical and polarization spectral interferometry", J. Opt. Soc. Am. B 15, 2331-2337 (1998). DOI

[7]. L. Lepetit, G. Cheriaux, M. Joffre, "Linear techniques of phase measurement by femtosecond spectral interferometry fo applications in spectroscopy", J. Opt. Soc. Am. B 12, 2467-2474 (1995). DOI

[8]. R. Trebino, Edt., Frequency-Resolved Optical Gating: The Measurement of Ultrashort Laser Pulses, Kluwer Academic, Massachusetts, USA (2000). DOI 
[9]. D. N. Fittinghoff, J. L. Bowie, J. N. Sweetser, R. T. Jennings, M. A. Krumbügel, K. W. DeLong, R. Trebino, I. A. Walmsley, "Measurement of the intensity and phase of ultraweak, ultrashort laser pulses", Opt. Lett. 21, 884-886 (1996). DOI

[10]. J. Garduño-Mejía, A. H. Greenaway, D. T. Reid, "Programmable spectral phase control of femtosecond pulses by use of adaptive optics and real-time pulse measurement", J. Opt. Soc. Am. B 21, 833-843 (2004).

\section{Introduction}

Femtosecond pulse shaping is typically achieved by different techniques which involve a phase or intensity modulator such as a liquid-crystal display (LCD) array [1], acousto-optic programmable dispersive filters (AOPDF) [2] and micro-machined deformable membrane mirrors (MMDM) [3]. A main limitation in the convergence speed on pulse shaping is related with the pulse characterization speed itself. Reported convergence refresh rates of pulse characterization are between $1-20 \mathrm{~Hz}$ applying different techniques $[4,5]$.

A suitable option to improve the refresh rate, sensitivity with a simple setup for femtosecond pulse characterization is spectral interferometry $[6,7]$. This method is very sensitive since depends on a linear response with the only condition that we must provide a reference pulse. The technique consists on resolving, in the frequency domain, the interferogram generated by the pulse, to be characterized, and its delayed (fixed) reference pulse in a spectrometer. Actual spectral phase information is retrieved by a Fourier transform, side band filtering and phase unwrapping process. To complete the computation, actual spectral phase of the reference pulse is subtracted. If the pulse reference is bandwidth limited (BL), spectral phase calculation of a shaped pulse is then a straight forward process.

In the present work we present a high speed femtosecond pulse characterization, based on spectral interferometry technique [7], with a refresh rate of $74 \mathrm{~Hz}$, to measure pulses from a shaper based on a MMDM.

\section{Experimental setup}

Experimental setup involves a Mach-Zender interferometer where a pulse is divided in two replicas: one is sent to the shaper and the other one is delayed and characterized with a home- made SHG-FROG $[4,8]$ to provide a reference pulse (Fig. 1(a)). At the output both pulses are recombined collinearly in a spectrometer $(0.1$ $\mathrm{nm}$ resolution, Ocean Optics HR2000+ series). The combination of the SHG-FROG, to measure the reference phase, and the spectral interferometry (SI), to retrieve the shaped phase, has been denominated as temporal analysis by dispersing a pair of light electric fields (TADPOLE) [9]. The main advantage of this technique, in addition to its good sensitivity, is that the reference phase needs to be measured just once if we assume that our femtosecond laser system (Coherent MIRA 900) remains stable during the experiment. The system has demonstrated very good stability, with no change of emission characteristics, during a couple of days.

The pulse shaper is based on a dispersionless design incorporating a 1200 lines/mm diffraction-grating and arranged in a $2 \mathrm{f}$ configuration with a deformable mirror situated at the Fourier plane of a $500 \mathrm{~mm}$ focal length concave mirror (Fig. 1(b)). Pulses from a selfmode-locked 200 femtosecond Ti:sapphire laser with a full width half maximum (FWHM) of 7.5 $\mathrm{nm}$ and centered at $826 \mathrm{~nm}$ (Coherent MIRA 900), were coupled into the shaper and dispersed to form a line spectrum extending across most of aperture of the mirror by adjusting the angle of the diffraction grating angle. Programming a chosen curvature onto the mirror surface produced a change in the spectral phase across the pulse spectrum. The MMDM mirror was fabricated by OKO Technologies and has a rectangular $11 \times 39 \mathrm{~mm}$ aperture, containing a 19-channel linear micro-machined deformable gold-coated membrane. With a minimum settling time of $2 \mathrm{~ms}$ and maximum deflection at the mirror center of $\sim 6 \mu \mathrm{m}$ the deformable mirror could produce a maximum spectral phase change of approximately \pm 40 radians, respect the laser central wavelength. 

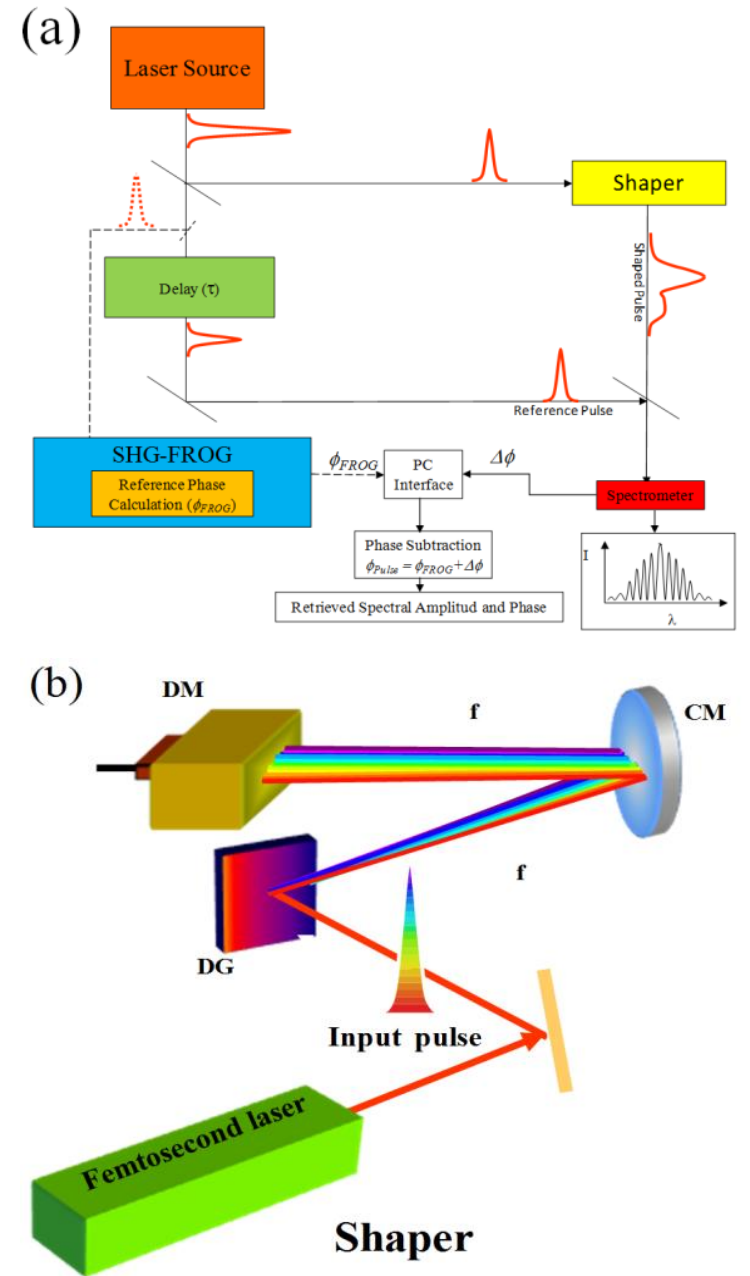

Fig. 1. Experimental and pulse shaper setup. In (a) the TADPOLE setup is presented and in (b) the pulse shaper based on a deformable mirror (DM), concave mirror with focal length of $f(\mathrm{CM})$ and a diffraction grating (DG).

The procedure to retrieve the actual pulse spectral amplitude and phase by using spectral interferometry is presented in Fig. 2.

An inverse fast Fourier transform (iFFT), at a fixed delay time $\tau$, is applied to the spectrogram. A side band is selected and filtered from the rest of the data. A fast Fourier transform (FFT) is applied and the difference spectral phase, $\Delta \phi(\omega)$, is retrieved by an unwrapping process. The actual pulse spectral phase, $\phi_{\text {Pulse }}(\omega)$, is estimated by adding the reference phase, $\phi_{F R O G}(\omega)$ and the difference spectral phase. With the actual spectral amplitude and phase the information about the pulse is complete.

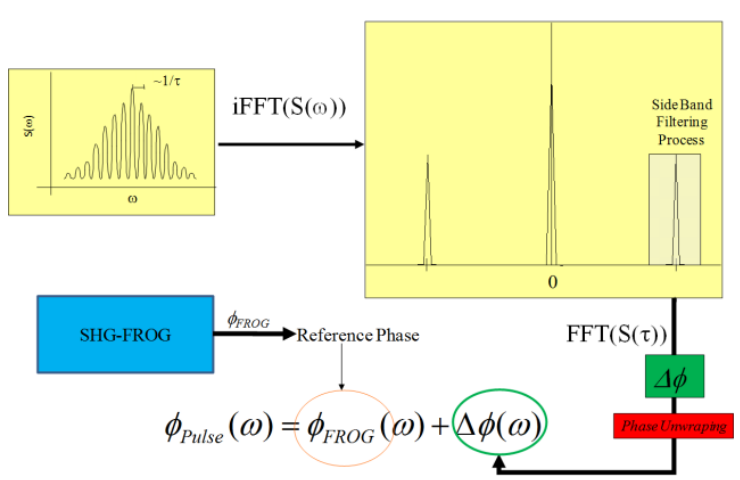

Fig. 2. Spectral interferometry phase retrieval procedure.

For practical purposes for instruments control and the data acquisition, the analysis and interface were performed with LabView TM. A snapshot of the Windows interface is showed in Fig. 3.

The pulse shaper interface includes the MMDM individual actuator voltage control sliders, spectrogram visualization, and retrieved spectral phase.

\section{Results}

Preliminary results demonstrate that the pulse characterization technique in combination with a fast speed spectrometer is able to retrieve the spectral amplitude and phase, in real time, at 74 $\mathrm{Hz}$ refresh rate in LabView. Since most of the driving algorithms for pulse design, in dynamicclosed-loop femtosecond pulse shapers are based on evolutionary algorithms, the fast acquisition and characterization rate of the shaped pulses represents a critical issue.

In the following Fig. 4 we present the retrieval of high order chirped phases, $\phi_{\text {Pulse }}(\omega)$, generated by the shaper. In the results, reference spectral phase, $\phi_{F R O G}(\omega)$, measured with a SHG-FROG, was already subtracted by following the procedure described in Fig. 2.

To test the shaper, in a closed loop, assisted by the fast rate spectral interferometry pulse retrieval, we define a flat spectral phase as a target. The fast repetition rate pulse characterization demonstrates to be a great support in the time convergence of the shaper. 


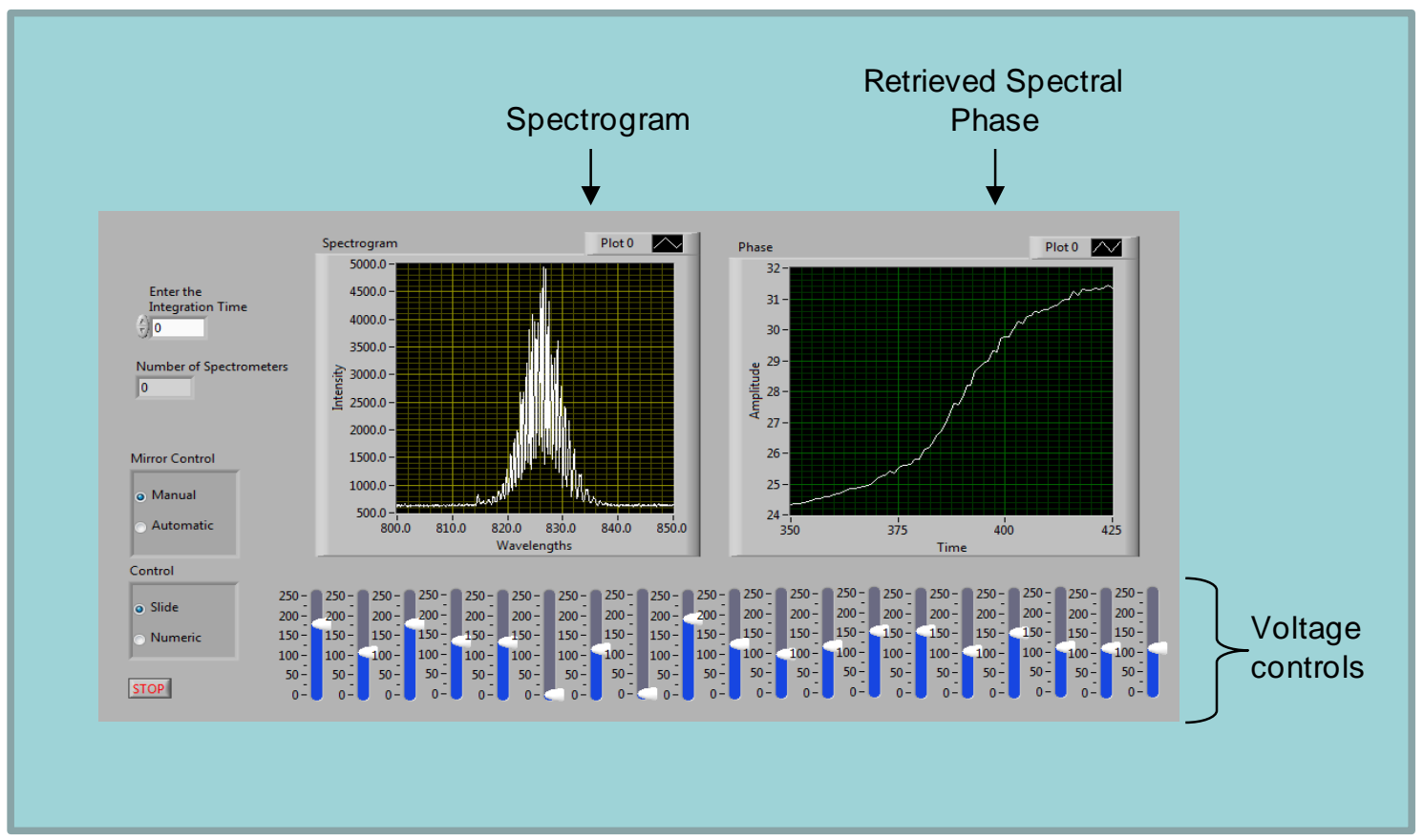

Fig. 3. Print screen of the MMDM based pulse shaper interface window: the pulse shaper interface showing the different components involved in the shaper operation including MMDM control, spectrogram visualization, and retrieved spectral phase.
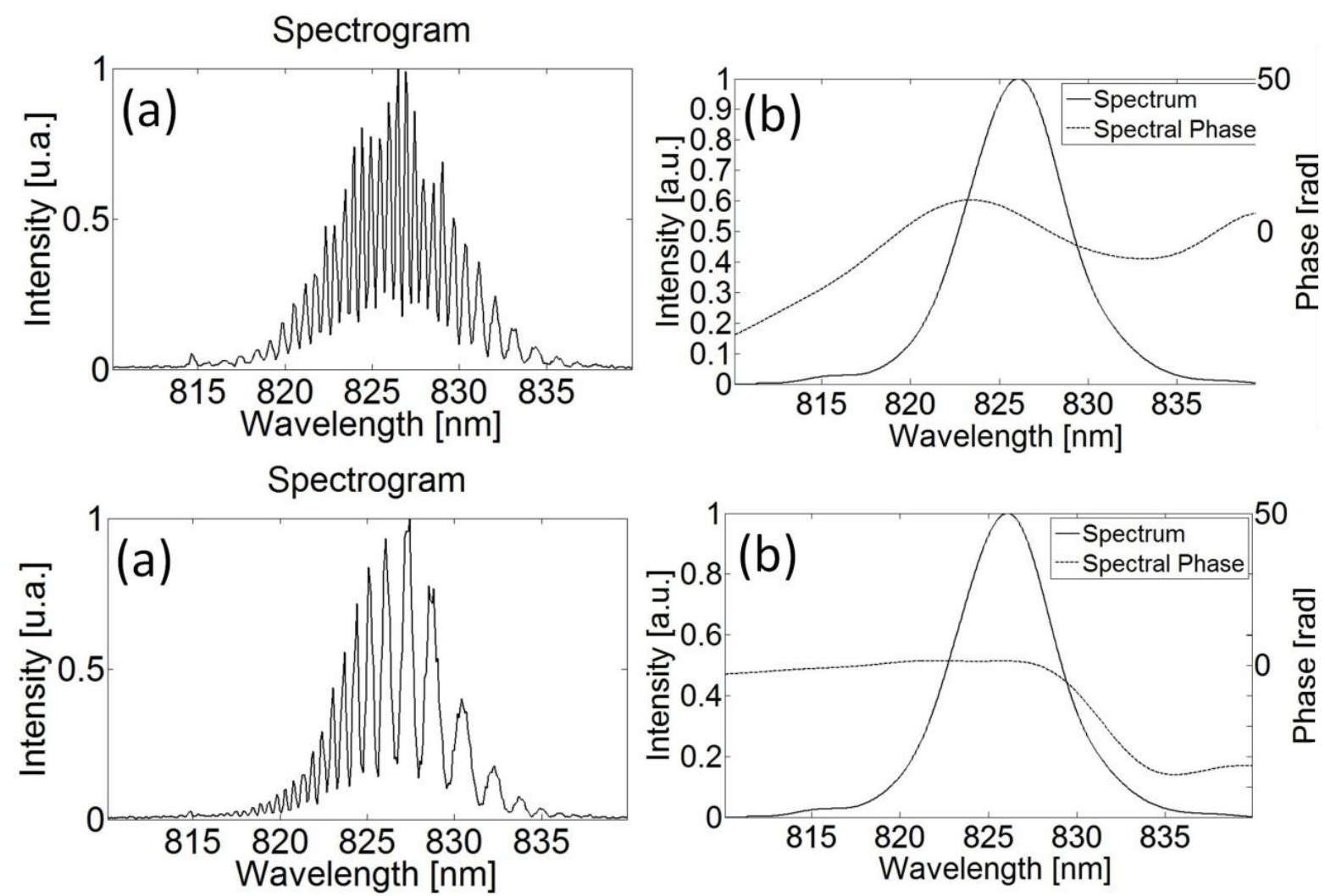

Fig. 4. High order spectral phases retrieved with spectral interferometry. In (a) the measured interferogram. In (b) the measured power spectrum and retrieved spectral phase. 


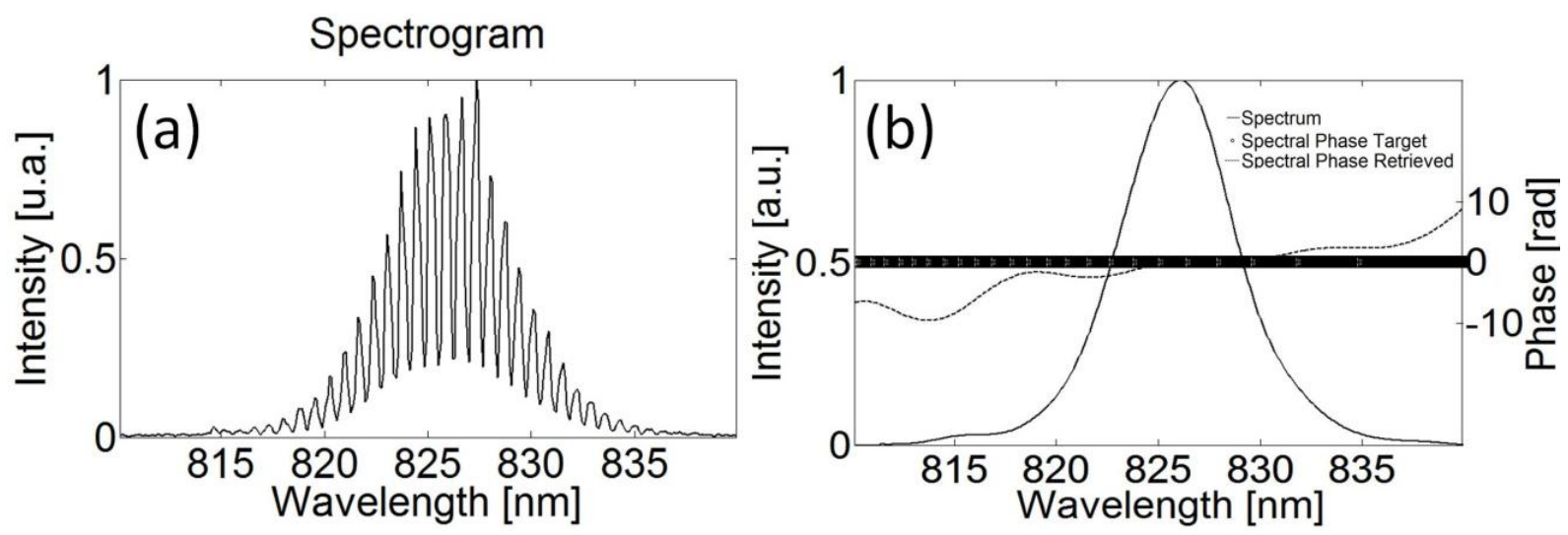

Fig. 5. Near Bandwidth Limited Spectral Phase along the main region of the power spectrum. In (a) the measured interferogram. In (b) the measured power spectrum (solid), retrieved spectral phase (dashed), target spectral phase (circles).

Spectral phase retrieval was achieved in a few seconds. The result is presented in Fig. 5. Near constant phase correspond to the surface mirror flat position. Mirror shape was previously measured with an interferometric method [10].

The driving algorithm, to achieve a target spectral phase $\phi_{\text {target }}$, is described in the flow chart in Fig. 6.

\section{Conclusions}

In the present work we demonstrate a high speed femtosecond pulse characterization, applying Spectral Interferometry technique, with a refresh rate of $74 \mathrm{~Hz}$, to measure pulses designed with a shaper based on a Micromachined Membrane Deformable Mirror (MMDM). As far as we know this is the fastest rate for femtosecond pulse characterization that has been reported to date. Although for practical purposes the instruments control and data acquisition were performed with LabView, fastest retrieval rates are expected by using $C^{++}$ or similar compilers.

Since most of the driving algorithms for pulse design are based on evolutionary algorithms, the fast acquisition and characterization rate of the shaped pulses represents a critical issue. The high speed refresh rate in the pulse characterization represents a great advantage for pulse shaping speed convergence. The spectral interferometry method represents an excellent alternative to be integrated in a closed loop femtosecond pulse shaper.

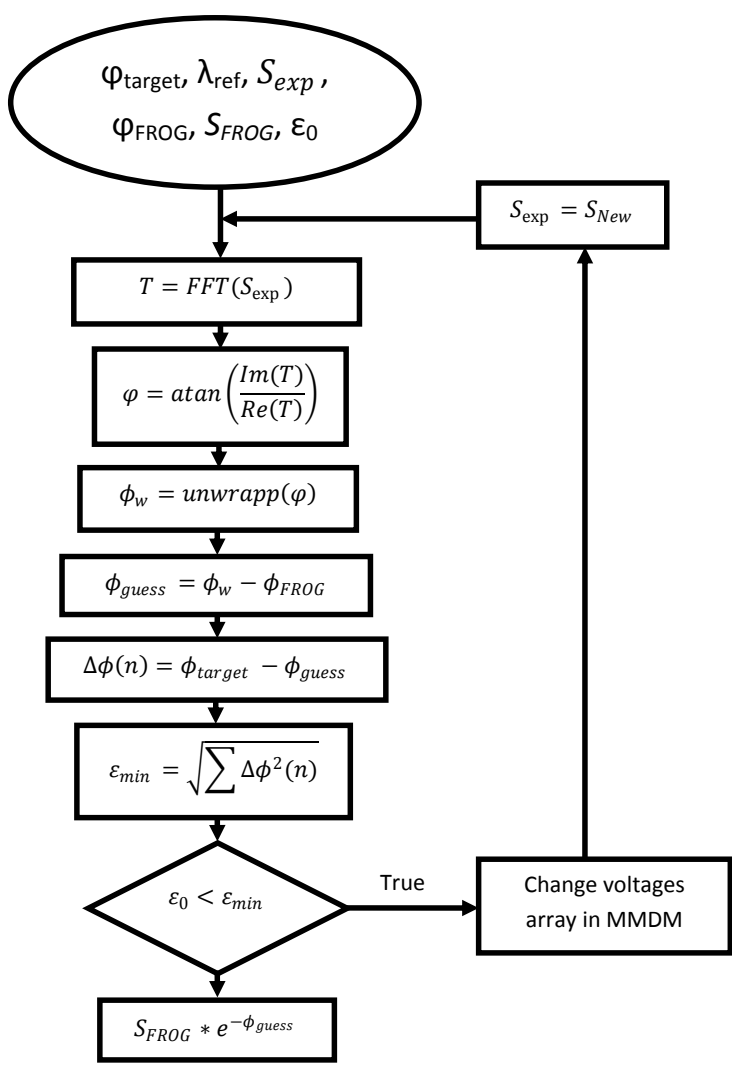

Fig. 6. Flow chart showing the algorithm used to determine the spectral phase, $\phi_{w}$, introduced by MMDM and corresponding actuator voltages necessary to achieve a target spectral phase $\phi_{\text {target }}$. The input variables includes the modulated spectrum, $S_{\text {exp }}$, the spectral amplitude and reference phase measured with the SHG-FROG system, $S_{F R O G}$ and $\phi_{F R O G}$, laser central wavelength, $\lambda_{\text {ref }}$, and fitness error $\varepsilon_{0}$. 
This characterization method is very sensitive since depends on a linear response, easy to setup with the only drawback that we must provide a reference pulse. Nevertheless, depending on the femtosecond laser source, the reference pulse could remain constant for long periods of time and then its characterization should be performed a couple of times every day.

\section{Acknowledgements}

The authors gratefully acknowledge sponsorship of this work by DGAPA-UNAM. PAPIIT project: IN113809, IB101212, IACOD-I1100611, IN104112, BID-UNAM 98-B3-C-DGP-L00341077, CONACyT-Mexico project CB 131746. 